\title{
Le nom propre en débat au tournant du siècle (Whitney - Bréal - Saussure)
}

\author{
Pierre-Yves Testenoire \\ ERIAC, Université de Rouen \\ pytestenoire@yahoo.fr
}

Le nom propre s'est davantage imposé, depuis le $\mathrm{XIX}^{\mathrm{e}}$ siècle, comme objet d'étude des logiciens et des philosophes que des linguistes. Bien qu'un ensemble d'études récentes s'attache aux problèmes morphologiques ou syntaxiques - antonomase, modification...- qu'il suscite, il est indéniable que de John Stuart Mill à Georges Kleiber, la réflexion théorique sur le nom s'est principalement axée autour de la question de la référence. Dans l'histoire de cette approche logicienne, le tournant des $\mathrm{XIX}^{\mathrm{e}}$ et $\mathrm{XX}^{\mathrm{e}}$ siècles constitue certainement un des moments les plus riches, moment illustré par le débat qui opposa Frege et Russell. En 1892, Frege publiait Sens et Dénotation; en 1905, dans un article intitulé « On denoting », Russell lui répondait et proposait sa théorie des descriptions définies. Notre ambition est de démontrer qu'en marge de cette célèbre polémique, le nom propre faisait aussi l'objet d'un débat dans les écrits des linguistes de ce temps. De manière contemporaine, on le sait, la linguistique se constituait comme science autonome par l'élaboration progressive d'un point de vue général sur les problèmes du langage. Dans ce mouvement, trois acteurs vont nous intéresser : William Dwight Whitney, Michel Bréal et Ferdinand de Saussure. Notre choix n'est pas arbitraire : tous trois sont des comparatistes ayant chacun entrepris à des degrés divers d'exprimer des idées sur le langage dépassant l'approche strictement historique de leur temps. De plus, leurs œuvres principales - The life and growth of language (1875), L'Essai de sémantique. Science des significations (1897) et le Cours de linguistique général dispensé à Genève de 1907 à 1911 et dont des disciples tirèrent un livre en 1916 - nous permettent de couvrir ce vaste espace chronologique au cours duquel la linguistique s'est structurée. Nous entreprenons donc l'examen critique du traitement par chacun d'eux de cet objet linguistique si singulier qu'est le nom propre. Si ce parcours suit la chronologie en s'arrêtant successivement sur la pensée des trois linguistes, il sera surtout porté attention aux échos et aux réponses que l'on peut entendre des unes aux autres. En définitive, le nom propre nous intéressera ici dans la mesure où il constitue l'objet d'un débat éclairant les théories du signe et du sens proposées par ces linguistes.

\section{Le nom propre au cœur du conventionnalisme : Whitney}

Dans La vie du langage, publié en anglais en 1875 puis traduit par l'auteur en français en 1877, la question du nom propre intervient à trois moments de l'argumentation de Whitney. Ce sont ces trois moments que nous voulons examiner successivement.

\subsection{De Georges à garçon}

Dès les premières lignes de son ouvrage, Whitney définit le langage comme «l'expression de la pensée humaine $» .{ }^{1}$ Il place évidemment ainsi la conscience des sujets parlants comme moteur des faits de langage au cœur de sa pensée théorique. Une telle définition met également l'accent sur les rapports qu'entretiennent les éléments linguistiques avec les objets du monde dont ils seraient la représentation. Cet aspect se trouve illustré dans la description qui est donnée, au second chapitre, de l'apprentissage de la langue maternelle par l'enfant. Cet apprentissage consiste, selon le linguiste, en la découverte de la correspondance entre les noms et les objets qu'ils désignent et le perpétuel réajustement de ces correspondances. L'enfant se familiarise avec les noms singuliers avant de maîtriser les noms généraux : « Nous avons déjà remarqué combien les enfants commettent souvent cette erreur d'employer les mots de papa et de maman pour signifier homme et femme. [...] Un peu plus grand, l'enfant apprend à prononcer, 
par exemple, le nom de Georges, mais il découvre qu'il ne doit pas appeler Georges des êtres trèssemblables à celui auquel le nom appartient, et qu'il y a pour cela un autre mot, celui de garçon. $»^{2}$ Les noms à référents individuels apparaissent ainsi comme premiers dans la conscience des sujets parlants. C'est parce que les noms comme garçon désignent des classes d'objets qu'ils sont seconds dans l'apprentissage. Ainsi que l'exprimera très clairement Whitney un peu plus loin, «acquérir le langage c'est adopter des classifications. ${ }^{3}$ Derrière ces considérations, peut-être aura-t-on reconnu diverses notions reprises au Système de logique déductive et inductive de John Stuart Mill rédigé une trentaine d'années auparavant. En effet, la distinction entre «Georges» et "garçon» recouvre la première dichotomie qu'établit le philosophe dans son examen du nom, celle entre nom singulier et nom général. La spécificité de ce dernier consiste dans le fait de désigner une «classe » définie de la façon suivante : « une classe est la multitude indéfinie d'individus désignés par un nom général. ${ }^{4} \mathrm{~A}$ ce critère de la «classe » repris dans le clivage Georges / garçon, est adjointe l'idée d'un progrès linguistique. Chez Whitney, la complexité conceptuelle d'un nom apparaît dès lors proportionnelle à son étendue référentielle.

La question qui va nous intéresser à présent, dans notre aperçu sur la question du nom propre, est celle de savoir si, de même, Whitney fait sienne la division établie par Mill entre noms connotatifs et nonconnotatifs. Rappelons rapidement la position de Mill qui pointe la solitude radicale des noms propres : «Les seuls noms qui ne connotent rien sont les noms propres; et ceux-ci n'ont, à strictement parler, aucune signification. $\gg{ }^{5} \mathrm{Du}$ point de vue logique, les noms propres fonctionnent comme de simples étiquettes et se distinguent des noms tels que Soleil, Dieu qui, eux, sont connotatifs et dont l'unicité référentielle est, selon Mill, accidentelle. Ce que dit Whitney n'est en apparence pas très différent : «Les classes sont, sans doute, d'étendue fort différente. Il y en a même - comme soleil, lune, Dieu, monde - qui se réduisent à une unité. $\mathrm{Il}$ y en a d'autres, dans lesquelles les unités ont pour nous une telle importance que nous leur donnons, en plus, ce que nous appelons un nom propre : tels sont les personnes humaines, les animaux domestiques, les rues, les villes, et autres localités, les planètes, les mois, les jours de la semaine et ainsi de suite. ${ }^{6}$ La spécificité des noms propres comme instruments de dénotation est soulignée. Celle-ci est attribuée à l'importance qu'ils recouvrent pour les sujets parlants, importance déjà illustrée dans la primauté pour l'enfant de Georges sur garçon. Whitney paraît ainsi utiliser la grille d'analyse du nom élaborée par Mill. Quant à sa position sur la question tant problématique du sens des noms propres, il convient de suivre encore son exposé et tout particulièrement les modalités de cette « vie du langage ».

\subsection{La vie du nom propre}

Filant la métaphore du titre, Whitney consacre quatre chapitres à la croissance et au développement du langage. Les chapitres quatre et cinq portent respectivement sur le « changement de la forme extérieure des mots » et sur le "changement du sens des mots", les six et sept sur la disparition et la création de nouveaux mots et de nouvelles formes. Cette scission fondamentale du phonétique et du sémantique, pour utiliser un terme que Whitney n'emploie pas, est fondée sur la « nature du lien entre le sens et la forme, nature toute accidentelle et arbitraire. » Ainsi forme et sens suscitent deux espèces de changement, "chacune des deux suit sa proche marche et reconnaît ses propres causes. ${ }^{7}$ Cela dit, une certaine cohésion dans l'étude des évolutions linguistiques est recherchée. L'auteur reconnaît d'ailleurs que « tous les modes du développement linguistique se confondent et se mêlent tellement qu'il est impossible de discuter l'un, si brièvement que ce soit, sans toucher plus ou moins le sujet des autres. ${ }^{8}$ Sans doute estce par souci de cohésion que les mêmes exemples - priest (prêtre), bishop (évêque) - sont analysés dans les chapitres quatre et cinq. Aussi est-il remarquable que les noms propres apparaissent uniquement dans le cinquième chapitre sur le sens des mots et soient absents de la réflexion sur les changements phonétiques.

La difficulté de l'analyse des changements de significations apparaît clairement à Whitney dans ce chapitre, qui note: «Il est tout simplement impossible d'épuiser la variété des changements de significations qui ont eu lieu dans les mots. $"{ }^{9}$ Quelques mouvements principaux sont néanmoins 
dégagés : restriction et extension de sens, transfert de l'acception propre à l'acception figurée des mots, passage du concret à l'abstrait. Arrêtons-nous sur les deux premiers.

Voici l'exemple qui est donné pour illustrer ce double tropisme : «C'est le principe de l'extension du sens des mots, opposé à celui de leur restriction. Un nom acquis par spécialisation commence une carrière indépendante et finit par être chef de tribut. M. Miller doit son nom à la profession qu'il exerce et qui est celle de meunier, de mill (moulin). Il devient l'ancêtre commun de toute une postérité de Millers, qui héritent de son nom. Un d'entre eux devient le fondateur d'une secte qu'on appellera millerites, et ce nom deviendra aussi important dans la nomenclature théologique que celui d'Arius ou de Nestorius. $»^{10}$

Dans la perspective diachronique adoptée ici, les glissements du nom propre au nom commun puis du nom commun au nom propre deviennent exemplaires de ces transferts de signification. C'est par spécialisation, comprendre par restriction de sens, que le nom commun (mill) devient nom propre (Miller) ; inversement, si millerites est issu de Miller c'est par extension de sens ainsi que le rend explicite la notation de l'importance pris par le nom dans la nomenclature. Dans le schéma proposé par Whitney, noms propres et noms communs constituent ainsi les deux pôles opposés de la charge sémantique. Le nom propre se conçoit alors comme l'élément linguistique à la signification minimale, ou dans un point de vue dynamique, comme le moment de la restriction de sens maximale.

On aperçoit clairement ce qui sépare une telle conception de celle de Mill. Et on goûtera l'ironie, sans doute involontaire, du linguiste américain choisissant pour sa démonstration le nom même de celui dont il se démarque. Miller reste, même dans ce qu'il est de plus ténu, un nom connotatif et dès lors participe pleinement de la circulation diachronique, la vie, dit Whitney, de la langue. A tout moment, le nom propre peut intégrer ces mouvements de restriction et d'extension de sens, aperçus de «l'infinie variété ${ }^{11}$ des changements phonétiques. Objet linguistique pertinent, le nom propre va une dernière fois se retrouver au cœur de la réflexion théorique de La vie du langage.

\subsection{Le nom propre comme paradigme}

Le chapitre huit intitulé «comment se créent les mots» vient conclure l'examen de l'évolution des langues. Dans cette quête sur « la confection originelle des noms », celle des noms propres est abordée en premier lieu puisqu'elle est, écrit le linguiste, «très-facile à observer» et qu'elle «se fait au grand jour $\gg .^{12}$ Son modèle en est l'acte de baptême du nouveau né, acte uniquement gouverné par le choix des parents nomenclateurs et des habitudes de la société dans laquelle ils vivent. Whitney donne ensuite l'exemple des savants donnant un nom à leur découverte. Sans surprise les concepts mis en principe à l'origine lexicale sont ceux de conscience - «tout cela se fait sciemment $»^{13}$ - et d'usage. On l'aura reconnu, le modèle proposé ici est celui que propose Hermogène dans le Cratyle. Au nomothète divin cratyléen sont substitués des nomenclateurs humains dont la volonté et la conscience sont soumises aux jeux des normes sociales. A cet axiome conventionnaliste s'adjoint ici une conception de la langue comme une nomenclature d'objets et d'individus. Dans ce schéma de pensée, le nom propre sert alors de paradigme pour penser les autres créations lexicales. Celles-ci s'accomplissent sur cette même trame, seul le degré de conscience des sujets baptiseurs varie : «Or dans ce fait très-simple, à des degrés différents et avec la conscience plus ou moins nette de son existence, se trouve impliqué le phénomène de la confection des mots avec toutes ses variétés. S'il n'en était pas ainsi, le langage se composerait de deux parties discordantes : une partie serait d'une manière, une partie d'une autre. $»^{4}$

Conséquence de l'approche de Whitney : entre le nom propre et les autres formes lexicales, il n'y a pas de différence de nature mais de degré. La contradiction qu'impliquerait l'existence de deux modes de production au sein du langage en est l'argument indépassable.

Sarah Leroy et Valeria Muni Toke, dans une étude récente, ont relevé dans La vie du langage « un avantgoût de la position saussurienne, qui sort le nom propre du système de la langue. ${ }^{15}$ Outre que la position saussurienne est, comme nous le verrons, plus ambiguë qu'il ne l'est dit, la phrase sur laquelle s'appuie cette interprétation mérite que l'on s'y arrête. Au sujet de l'imposition des noms d'individus évoquée plus haut, Whitney remarque : "Ceci semble n'avoir rien de commun avec les faits de langage ». Mais 
aussitôt il ajoute : «Toutefois, ce n'est pas toujours le cas : le nom propre, Jules, a fait que le septième mois de l'année s'appelle chez nous juillet; le surnom de César a donné leur titre aux chefs de deux grands peuples, l'Allemagne et la Russie (Kaiser, czar) ; ». ${ }^{16}$ L'exclusion du nom propre du domaine de la langue est donc, on le voit, toute relative. C'est de l'évolution historique, seul point de vue adopté dans La vie du langage, dont le nom propre reste parfois extérieur. Parler ainsi de "système de la langue " dans l'acception synchronique que cette expression recouvre aujourd'hui semble ainsi inadapté au sujet de la pensée de Whitney. Mais surtout le refus explicite des «deux parties discordantes » au sein du langage invalide une telle lecture. On a vu qu'était récusé le clivage entre nom propre et nom commun qu'impliquait, sur le plan logique et sémantique, la théorie de Mill. Ce même clivage sur le plan linguistique n'est pas plus accepté au motif de l'intégration, à tout moment possible pour les besoins de la communication, des noms propres à la circulation de la langue, et dont Whitney multiplie les exemples : Miller - millerites, Jules - juillet... Ainsi non seulement la création lexicale fonctionne comparativement qu'il s'agisse de nom propre ou de tout autre vocable, mais bien plus l'impositio nominum est érigée en paradigme. Le modèle du nom sert la conception référentialiste de Whitney car, de même que l'enfant existe avant d'avoir un nom, explique-t-il, de même ce qu'il appelle les idées, soit les objets moins directement perceptibles à notre conscience, préexistent au langage. ${ }^{17}$ La question du nom propre n'est pas aussi annexe qu'elle ne le semblait au premier abord pour la conception du langage de Whitney, conception « adamique » comme la qualifiera Saussure. Mais n’anticipons pas.

\section{Des substantifs par excellence : Bréal}

Michel Bréal partage avec Whitney le souci d'affirmer le caractère historique des sciences du langage. Pour cela, l'un et l'autre s'attachent à dissiper les conceptions organicistes des langues telles qu'elles furent développées par un certain nombre de savants tout au long du XIX ${ }^{\mathrm{e}}$ siècle. ${ }^{18}$ Mais là où Whitney est encore redevable de ces vues dans la terminologie métaphorique qu'il continue d'employer - vie, croissance... -, Bréal prône une intransigeance absolue : «On nous a dit que les mots naissaient, se livraient à des combats, se propageaient et mouraient. Il n'y aurait aucun inconvénient à ces façons de parler s'il ne se trouvait des gens pour les prendre au sens littéral. ${ }^{19}$ La remarque, il est vrai, vise explicitement La vie des mots de Darmesteter, mais elle aurait pu tout aussi bien s'adresser au linguiste américain. Whitney serait-il alors un des interlocuteurs de l'Essai de Sémantique? Une telle lecture a quelques arguments pour elle. On peut par exemple remarquer que les chapitres dix à treize de l'Essai de Sémantique reprennent dans l'ordre les quatre tendances, résumées ci-dessus, du changement de sens des mots développées dans le cinquième chapitre de La vie du Langage. ${ }^{20}$ Mais c'est surtout le développement sur les noms propres dans le chapitre «comment les noms sont donnés aux choses » qui apparaît comme une réponse à celui de Whitney intitulé « comment se créent les mots ».

Le passage commence ainsi : «On a soutenu que les noms propres, comme Alexandre, César, Turenne, Bonaparte, formaient une espèce à part et étaient situés en dehors de la langue $»{ }^{21}$ Qui le pronom impersonnel désigne-t-il ? Faut-il voir ici une allusion à la théorie des noms propres vides de sens défendue par Mill ? Il est vrai que Bréal connaissait l'œuvre du philosophe puisqu'il le cite un peu plus loin dans l'Essai de Sémantique. ${ }^{22}$ Cependant les trois arguments donnés en faveur de cette thèse incitent à chercher dans une autre direction : «Pour cette catégorie le sens étymologique n'est d'aucune valeur ; de plus, ils passent d'une langue à l'autre sans être traduits; enfin ils suivent généralement les transformations phonétiques d'une marche plus lente. $\gg{ }^{23}$ Les idées exprimées ici ne sont guère originales. Elles ont été développées à plusieurs reprises dans le cadre du débat millénaire sur la nature de la dichotomie grammaticale nom propre - nom commun. ${ }^{24}$ Ainsi le constat de la marche phonétiquement plus lente du nom propre se trouve déjà chez Leibniz et de nouveau à l'époque de Bréal, chez Max Müller par exemple. ${ }^{25}$ Bréal rejette succinctement ces arguments en arguant leur caractère relatif puisque les mêmes remarques s'appliquent tout aussi bien, selon lui, à d'autres éléments du lexique : noms de dignité, de fonctions... Quant à l'oubli du sens étymologique, il est le principe même des lois intellectuelles que l'Essai de sémantique entreprend de dégager. ${ }^{26}$ Et le linguiste d'affirmer : « entre les noms propres et les noms communs il n'y a qu'une différence de degré $\gg .^{27}$ 
Un autre type de différence, non plus grammaticale mais sémantique, va faire l'objet d'un second paragraphe: «La différence avec les noms communs est une différence toute intellectuelle. Si l'on classait les noms d'après la quantité d'idées qu'ils éveillent, les noms propres devraient être en tête, car ils sont les plus significatifs de tous, étant les plus individuels. Un adjectif comme augustus en devenant le nom d'Octave, s'est chargé d'une quantité d'idées qui lui étaient étrangères. D'autre part, il suffit de rapprocher le mot César, entendu de l'adversaire de Pompée, et le mot allemand Kaiser, qui signifie « empereur », pour voir ce qu'un nom propre perd en compréhension à devenir nom commun. D'où l'on peut conclure qu'au point de vue sémantique les noms propres sont les substantifs par excellence. ${ }^{28} \mathrm{La}$ position adoptée ici s'oppose diamétralement à celle de Whitney. Les pôles sémantiques que constituaient les noms propres et les noms communs dans La Vie du langage se trouvent en effet renversés et, comme pour le souligner, Bréal reprend les exemples d'empereurs romains déjà utilisés par Whitney. Ici, la forte charge sémantique attribuée au nom propre est présentée comme conséquence de son caractère individuel. En corrélant ainsi sémantisme et étendue référentielle, l'Essai de sémantique s'inscrit dans une tradition logico-grammaticale ancienne. L'opinion exprimée apparaît alors comme une nouvelle application de ce qu'il est convenu d'appeler la loi de Port-Royal selon laquelle l'extension d'un terme varie en raison inverse de sa compréhension. ${ }^{29}$

$\mathrm{Au}$ sujet de cette conception des rapports inversement proportionnels de l'extension et de la compréhension, ou pour le dire autrement, de la désignation et de la signification, il convient de noter, comme l'a fait Sylvain Auroux à propos des logiciens de l'époque classique, le « caractère quantitatif de la théorie, qui correspond à une véritable mathématisation de la théorie de la référence $»{ }^{30}$ La remarque s'illustre pertinemment bien dans ce passage de l'Essai de sémantique où s'exprime à l'aide de l'adverbe « plus » et en terme de «quantité d'idées » la relation sémantique du nom propre et du nom commun. Soulignons ensuite la cohérence de la pensée de Bréal car la position adoptée dans le premier paragraphe, position que nous qualifierons de péripatéticienne par son refus de la séparation des noms propres et communs en deux catégories grammaticales distinctes, s'éclaire à la lecture du second. Cette idée d'un continuum grammatical sert la conception d'un continuum référentiel dont noms propres et noms communs seraient les points extrêmes. En d'autres termes, le nom propre ne peut être accepté comme hypostase du nom à son degré d'étendue référentielle minimale que s'il est inféodé à la catégorie générale du nom comme le propose la doctrine classique. ${ }^{31}$ Et c'est sur ce point qu'en dépit de leur opinion exactement inverse concernant la charge sémantique du nom propre, le traitement de Whitney et de Bréal se rencontrent. Outre leur incapacité commune à penser une séparation autre que de degré entre noms propres et noms communs, la même proposition les réunit: les noms propres sont les substantifs par excellence. Cette affirmation, nous l'avons vu, doit s'entendre différemment chez l'un et chez l'autre. Pour Bréal, c'est la conséquence de l'acceptation de la loi de Port Royal tandis qu'il s'agit, chez Whitney, $\mathrm{du}$ fondement même d'une vision de la langue comme nomenclature. Cette excellence contraste singulièrement avec la place qu'occupe le nom propre dans la réflexion linguistique de Ferdinand de Saussure.

\section{Qu'en est-il du nom propre chez Saussure ?}

Les théories générales proposées par Whitney et par Bréal sont certainement celles dont la pensée linguistique de Saussure s'est le plus imprégnée, pour mieux s'en démarquer et les dépasser. C'est en tout cas en réaction à leurs idées que furent écrits les deux textes de linguistique générale les plus aboutis que nous possédions de la main du maître genevois : les notes pour un article d'hommage à Whitney de la fin de l'année 1894 et les Notes Item rédigées, nous y reviendrons, en réponse à l'Essai de sémantique de Bréal certainement dans les dernières années du siècle. C'est en raison de cette connaissance historiquement attestée de ces œuvres, laquelle se double d'une connaissance personnelle de leurs auteurs, que nous poursuivons notre étude en nous intéressant à la théorie saussurienne. ${ }^{32}$ Un premier constat s'impose : sur le débat relatif au nom propre opposant Whitney et Bréal, Saussure observe un silence intriguant. 


\subsection{Un silence intriguant}

La question du nom propre est absente du Cours de linguistique générale. Cela a déjà été remarqué, le nom propre constitue un hors champs de la théorie saussurienne du signe. ${ }^{33}$ La cause semble entendue : signe au signifié hautement problématique et singulier, si ce n'est inexistant, le nom propre est rétif à la notion de valeur et s'exclut de ce fait du système de la langue. Comme, de plus, la dichotomie langue parole proposée semble rendre théoriquement possible une telle exclusion, on peut penser que Saussure, par le silence observé dans le Cours, acte le caractère non sémiologique du nom propre. La question mérite pourtant, nous semble-t-il, d'être réexaminée. Il convient, pour la compréhension de la pensée du linguiste genevois, de ne pas se limiter au seul Cours de linguistique générale composé par Bally et Séchehaye et, pour ce faire, d'exploiter les textes autographes que la philologie saussurienne s'emploie depuis un demi-siècle à faire connaître. La connaissance de ces documents permet de mesurer le contraste entre l'importance qu'y occupe la problématique du nom propre et son absence dans l'enseignement dispensé par le linguiste de 1907 à 1911.

Arrêtons-nous encore un peu sur le Cours. Et nuançons quelque peu notre propos : le nom propre intervient à un unique moment dans l'enseignement de linguistique générale, un moment qui ne suscite habituellement guère l'intérêt. Il s'agit du premier cours (1907) où Saussure ne livre pas encore sa théorie générale de la langue mais dresse un examen critique de la linguistique historique de son temps et aborde la question des évolutions phonétiques. Au cours du long développement consacré à l'analogie, une remarque va nous intéresser, une remarque dont le Cours de linguistique générale conserve une trace dans sa troisième partie intitulée "Linguistique diachronique »: «Le seules formes sur lesquelles l'analogie n'ait aucune prise sont naturellement les mots isolés, tels que les noms propres spécialement les noms de lieux (cf. Paris, Genève, Agen, etc.), qui ne permettent aucune analyse et par conséquent aucune interprétation de leurs éléments ; aucune création concurrente ne surgit à côté d'eux. ${ }^{34}$ Signalons d'emblée que c'est par le traitement de la question des changements phonétiques qu'est relevée la spécificité des noms propres. La réflexion linguistique de Saussure rencontre ainsi le nom propre par la voie où précisément Whitney, on l'a vu, s'abstenait de l'aborder. Mais surtout il n'est plus question, comme chez Whitney de glissement par extension ou restriction de sens, ni d'une simple marche phonétique plus lente comme le notait Bréal. L'impossibilité d'une analyse morphématique des noms propres les exclut de ce « principe général des créations de la langue » ${ }^{35}$ qu'est, pour Saussure, l'analogie. La conviction de cet isolement linguistique si radicalement exprimée ${ }^{36}$ peut sembler aujourd'hui banale. D'un point de vue historiographique, elle ne semble pourtant n'avoir été acquise que parce que la question du nom propre a été, depuis le début des années 1900, au cœur des travaux scientifiques de Ferdinand de Saussure. De 1900 à 1904, en effet, toutes ses communications scientifiques sont consacrées à l'étude des toponymes de la région genevoise. ${ }^{37}$ La même sollicitude pour le nom propre en diachronie se retrouve, ces mêmes années, dans les recherches menées sur les légendes germaniques. Loin de se réduire à une simple étude sur les éléments historiques dans les récits tels que La chanson des Nibelungen ou les sagas scandinaves, ces travaux révèlent une quête de nature sémiologique sur le fonctionnement des symboles que constituent aux yeux de Saussure les personnages légendaires. Dans la formation de ces figures, le nom propre joue un rôle parmi d'autres :

« C'est dans cet esprit général que nous abordons une question de légende quelconque, parce que chacun des personnages est un symbole dont on peut varier, - exactement comme pour la rune -, a) le nom, b) la position vis-à-vis des autres - c) le caractère, d) la fonction, les actes. Si un nom est transposé, il peut s'ensuivre qu'une partie des actes sont transposés, et réciproquement, ou que le drame tout entier change par un accident de ce genre. $\rangle^{38}$

Plus loin, Saussure souligne que cette mutabilité du nom d'individu est spécifique à la vie sémiologique des récits légendaires : «Ici note sur les éléments constitutifs d'un être légendaire. Le nom n'a ni plus ni moins d'importance que tout autre côté. Il n'est pas comme chez un individu vivant une étiquette sur la personne, mais au même rang que les autres choses. ${ }^{39}$ Enfin il est notoire qu'en développant à partir de 1906 l'hypothèse d'une pratique anagrammatique dans la poésie antique, les mots que Saussure essaye de lire dans l'épaisseur des textes et qu'il appelle les «mot-thèmes» sont presque toujours des noms propres. $^{40}$ Il apparaît ainsi que, durant les années qui précédent les trois cours de linguistique générale, le 
nom propre s'impose comme objet central de la recherche saussurienne qu'elle soit historique, légendaire ou poétique. Toponymes défiant les lois des changements phonétiques, noms d'individus ondulant au gré de la transmission des légendes, ou distillant ses sonorités au sein des poèmes : Saussure aura eu maintes occasions de réfléchir à la radicale singularité de son objet. C'est pourquoi il rompt avec les positions que nous avons vues développées chez Whitney ou Bréal. «Mots isolés », «étiquette sur la personne » : on ne saurait forclore de manière plus absolue ces noms pour leur fonction dénotative. De nos trois linguistes c'est paradoxalement celui dont la connaissance de l'oeuvre de Mill est la plus incertaine qui semble s'approcher le plus de sa conception.

Pour résumer, à partir du début $\mathrm{du} \mathrm{XX}^{\mathrm{e}}$ siècle, le nom propre n'apparaît plus dans les écrits de linguistique générale de Saussure et se trouve relayé dans les recherches paralinguistiques périphériques dont il semble curieusement constituer le point commun. La raison de ce puissant clivage dans l'œuvre saussurienne est à lier au traitement théorique du problème de la référence. A ce sujet, la conviction que le langage ne saurait en aucun cas être une nomenclature d'objets constitue l'une des idées les plus anciennes, les plus souvent et les plus fortement répétées par Saussure. ${ }^{41}$ Depuis les premiers textes de linguistique générale, il ne cesse de fustiger cette conception du langage qu'il qualifie d'adamique, et écrit par exemple au début des années 1890 : «Il est malheureux 〈certainement〉 qu'on commence par y mêler comme un élément primordial 〈cette donnée〉 des objets désignés, lesquels n’y forment aucun élément quelconque. ${ }^{42}$ Les objets extralinguistiques n'ont pas leur place dans une théorie générale des langues. C'est la même position que développera le Cours de linguistique générale, même si l'on sait, depuis le célèbre article d'Emile Benveniste, qu'y affleure ça et là «le recours inconscient et subreptice à un troisième terme $\gg .{ }^{43} \mathrm{C}$ 'est au cœur même de l'élaboration, à l'aide du concept de valeur, d'une théorie de la signification purement intralinguistique que Benveniste pointe la confusion du référent et du signifié : «En réalité Saussure pense toujours, quoiqu'il parle d'«idée », à la représentation de l'objet réel et au caractère évidemment non nécessaire, immotivé, du lien qui unit le signe à la chose signifiée. ${ }^{44}$ Plus récemment Simon Bouquet, en prenant en compte les textes manuscrits, a relevé une oscillation de la pensée saussurienne sur la question de la référence. ${ }^{45}$ Vis-à-vis de la version forte, c'est-à-dire celle excluant l'objet externe du signe et par là même de la théorie de langue, qu'ont adoptée les éditeurs du Cours de linguistique générale se découvre aussi une version douce. C'est cette version davantage ouverte à l'objet qui va nous intéresser pour la question du nom propre.

\subsection{De l'onymique}

La principale manifestation de cette version se trouve dans ce qu'il est convenu d'appeler les Notes Item. Ces notes manuscrites non publiées du vivant de Saussure constituent, dans une facture formelle et terminologique bien différente de celle des Cours, une première élaboration de la théorie du signe linguistique. Bien qu'aucune certitude ne puisse être acquise quant à leur datation exacte, elles semblent avoir été écrites en réaction à la lecture de l'Essai de sémantique de Bréal. Son nom apparait d'ailleurs explicitement dans l'une d'entre elles où Saussure examine le concept d'ellipse. "Pourquoi parler d'ellipse (comme Bréal) » feint-il de s'interroger, alors que «les signes sont toujours adéquats à ce qu'ils expriment $?^{46}$ C'est évidemment la thèse de Bréal - «le langage désigne les choses d'une façon incomplète et inexacte ${ }^{47}$ - qui est visée. C'est donc avec cette visée polémique vis-à-vis de son ancien maître, qu'il faut également lire le traitement de la question du nom propre qui intervient un peu plus loin. Ce texte, exemplaire de cette ambiguité saussurienne quant à la référence et antérieur à la partition du nom propre dans ses travaux, est en grande partie lacunaire. Nous le citons in extenso dans l'édition d'Engler :

«† Item. Dès qu'il est question quelque part de la langue, on voit arriver le mot et le sens (ou le signe et le sens) comme si c'était ce qui résume tout, mais en outre toujours des exemples de mot comme arbre, pierre, vache, 〈comme Adam donnant des []〉, c'est-à-dire ce qu'il y a de plus grossier dans la sémiologie : le cas où elle est (par le hasard des objets 〈qu'on choisit pour être〉 désignés) une simple onymique, c'est-à-dire, car là est la particularité de l'onymique dans l'ensemble de la sémiologie, le cas 
où il y a un troisième élément incontestable dans l'association psychologique du sème, la conscience qu'il s'applique à un être extérieur 〈qui devient [b.]〉 assez défini pour échapper à la loi générale du signe.

$\dagger$ Item. Quoique nous voulions aborder le moins possible le côté idéologique du signe, il est bien évident que si les idées de toute espèce offraient une fixité []. - Fixité seulement obtenue par les noms géographiques. /[2]

$\dagger$ L'idée invariable et influctuable pouvant être considérée comme chose chimérique, les sèmes géographiques 〈et les noms propres〉 font exception en ce que... Plus exactement : [] La seule chance pour un []. (L'idée dans tout cela est la question de savoir si de même qu'un aposème persiste hors du sème, de même un aposème intellectuel pourrait quelque part être constaté. Certain que dans 'Rhône' il y a pour ainsi dire deux aposèmes courant parallèlement. Mais au fond rien de semblable possible, puisque si on avait changé le nom du Rhône, il n’y aurait plus le même sème, et dès lors inutile de discuter des aposèmes, et ainsi meilleure preuve que le sème a sa base fondamentale dans le signe matériel choisi.) ${ }^{48}$

Tout commence avec la figure d'Adam et la critique, fréquente chez Saussure nous l'avons dit, de la langue comme nomenclature. L'erreur à l'origine de cette conception est de prendre comme modèle pour penser la langue ce qui n'est en fait qu' «un cas particulier »: les cas où les référents des signes linguistiques sont particulièrement prégnants dans la conscience des sujets parlants. Remarquons que ce qui est décrit ici correspond exactement à la démarche que nous avons mise au jour chez Whitney. A ce cas particulier, le «plus grossier dans la sémiologie », Saussure donne le nom d'onymique. Arbre, pierre, vache viennent dans un premier temps l'illustrer ${ }^{49}$ mais très vite ce sont les noms géographiques qui s'imposent, dans la démonstration, comme archétypes de ces signes au fonctionnement particulier. Car, soulignons-le, les « sèmes » - c'est-à-dire ce que Saussure appellera les signes dans le Cours - qui relèvent de l'onymique font partie de la sémiologie. Puisque ce qui les distingue des autres signes est l'importance de leur « troisième élément » - le «troisième terme » de Benveniste - c'est bien qu'ils en ont déjà, comme les autres, deux. Le principe de la bifacialité du signe, dont les Notes Item constituent la première théorisation, s'applique donc aux noms propres.

Par ailleurs, on lit dans une autre Note item découverte plus récemment mais certainement contemporaine de celle qui nous intéresse :

«Item. Les réalités sémiologiques ne peuvent à aucun moment se composer [ ]

De sorte que vous êtes placé depuis le début devant ce dilemme :

-ou bien vous vous occuperez seulement du mouvement de l'index, ce dont vous êtes libre, mais alors il n'y a ni sémiologie ni langue dans ce que vous explorez;

-ou bien, si vous voulez faire de la sémiologie, vous serez obligé non seulement [ ]. " ${ }^{50}$

L'alternative entre «sémiologie » et «mouvement d'index » ne saurait être exprimée de manière plus catégorique. Les noms propres inclus dans le champs sémiologique sous la bannière spéciale de l'onymique ne relèvent donc pas de ce mouvement d'index, ne se réduisent pas à leur unique valeur dénotative comme le voudrait Mill. Certaines hypothèses suscitées par le silence de la théorie du signe développée dans le Cours de linguistique générale sur cet aspect se dissipent alors : pour Saussure, le nom propre n'est pas vide de sens, ou privé de signifié comme on a pu le dire. Signe biface, relevant de la langue et de la sémiologie, sa spécificité fait toutefois qu'il «échappe à la loi générale du signe ». Cette spécificité explique sans doute le silence des Cours sur cette question.

\subsection{De la parasémie}

De l'aveu même de Saussure, l'irruption du nom propre dans le cours même de son raisonnement l'amène sur un terrain où il ne comptait pas aller : «le côté idéologique du signe ». La fixité référentielle des noms géographiques suscite, pour la première fois, dans la note reproduite ci-dessus, un questionnement relatif à l'existence d'une diachronie du sens. Saussure avait précédemment créé le concept d'aposème, cette unité phonique - «L'aposème est l'enveloppe du sème $»^{15}$ - permettant de concevoir l'identité à travers le temps de deux éléments linguistiques: "Diachroniquement la question : est-ce le même mot? signifie uniquement est-ce le même aposème? ${ }^{52}{ }^{5}$ Sur le modèle de cette unité physique diachronique, peut-on 
supposer une unité diachronique de signification? Sans cette fixité référentielle des noms propres, et tout particulièrement celle des noms géographiques, il est difficile d'envisager l'hypothèse de l'aposème intellectuel. La réponse saussurienne est toutefois négative : l'idée indépendante du signe ne relève pas de la linguistique. Bien plus, la signification est, pour Saussure, un phénomène synchronique puisque elle résulte du jeu contrastif des termes coexistant dans le système de la langue. Les Notes Item, on l'a dit, combattent la sémantique de Bréal. Celle-ci suppose une certaine indépendance du sens vis à vis du signe et du système dans lequel il se situe inacceptable pour le linguiste genevois. ${ }^{53}$ De ce point de vue, il est tout à fait remarquable que la réflexion sur le nom géographique suscite la première formulation explicite de la nature négative et oppositionnelle de la signification telle que la conçoit Saussure. C'est en effet à la suite de cet échec à suivre l'aposème intellectuel du Rhône que la notion de parasème se trouve définie :

«Item. Les parasèmes. Pour un mot quelconque 〈faisant partie de〉 la langue, un $\langle$ second $\rangle$ mot, même n'ayant avec le 〈premier〉 aucune 'parenté', est un parasème. 〈La〉 seule et simple qualité 〈du parasème〉 est de faire partie du même système psychologique de signes, de manière que si l'on trouve, après observation, 〈qu'un signe donné a sa complète existence hors des signes concurrents du système〉, 〈qu'il n'y a point d'importance à observer [b.]〉 pour un signe donné l'ensemble des signes concurrents, le mot de parasème devra tomber, et réciproquement 〈il devra〉 subsister si on constate qu'un mot n'est 〈point complètement $\rangle$ autonome dans le système dont il fait part[ie]. ॥ ${ }^{54}$

Dans la note précédente, Saussure avait évacué l'espoir que l'idée de «Rhône » puisse être suivie en diachronie : même pour les signes dont le référent laisse supposer une certaine fixité, une sémantique diachronique s'est révélée illusoire. L'invention du parasème apparaît comme la conséquence directe de ce constat. C'est l'inconsistance même de l'aposème intellectuel qui semble prouver la nécessité théorique du parasème. En proposant cette nouvelle notion, le linguiste reconnaît que le « coté idéologie du signe » ne peut s'appréhender que sur l'axe synchronique. De ce point de vue, la parasémie, entendue comme la coexistence des signes dans un système de langue, donne un premier aperçu du concept saussurien de valeur.

A la fin du troisième cours, on le sait, la signification d'un mot est présentée comme résultant de deux relations : la relation arbitraire, à l'intérieur du signe, de l'image auditive et du concept et la relation contrastive, à l'intérieur d'un état de langue, des signes entre eux. C'est à cette seconde relation qu'est donnée le nom de valeur. Dans une de ses dernières leçons, Saussure met en lumière la primauté paradoxale de la valeur dans la constitution du sens : «Quand on parle de valeur, on sent que cela devient ici synonyme de sens (signification), et cela indique un autre terrain de confusion (ici la confusion qui sera davantage dans les choses elles-mêmes). La valeur est bien un élément du sens, mais il importe de ne pas prendre le sens autrement que comme une valeur. C'est peut-être une des opération les plus délicates à faire en linguistique, de voir comment le sens dépend et cependant reste distinct de la valeur. $"{ }^{55}$ Le linguiste s'attache ainsi à démontrer que le jeu du signifié et du signifiant interne au signe est déterminé par celui, externe, des signes entre eux. Ainsi la signification, « contrepartie de l'image acoustique », est elle-même une valeur, "contrepartie des termes existant ». ${ }^{56}$ L'analyse saussurienne de la signification est ainsi entièrement fondée sur cette conception de la valeur, comme le prouve, à la fin du chapitre qui lui est consacré, cette définition du signifié : "Le mot n'existe pas sans un signifié aussi bien qu'un signifiant. Mais le signifié n'est que le résumé de la valeur linguistique supposant le jeu des termes entre eux, dans chaque système de langue. $\gg{ }^{57}$

Lors de la rédaction des Notes Item, le linguiste ne disposait pas encore d'une vision aussi cohérente que celle qu'il livrera dans ses Cours de linguistique générale. La parasémie ne constitue certes pas une explication structurée de la production sémantique. Elle préfigure néanmoins, par sa dimension exclusivement synchronique, le concept de valeur développé dans le Cours. "La valeur ou la contemporanéité, c'est synonyme. $\rangle^{58}$, dira Saussure en 1911. Dans la terminologie des Notes Item, contemporanéité et parasémie, le sont également. Cette nouvelle notion n'est toutefois formulée, dans la note reproduite ci-dessus, que comme une hypothèse. La pertinence théorique du parasème, écrit Saussure, dépend du degré d'interdépendance des signes entre eux car " si l'on trouve, après observation, 〈qu'un signe donné a sa complète existence hors des signes concurrents du système〉, [...] le mot de 
parasème devra tomber, et réciproquement 〈il devra〉 subsister si on constate qu'un mot n'est 〈point complètement〉 autonome dans le système dont il fait part[ie]. " L'alternative posée ici rejoint celle soulevée par la problématique du nom propre. Ce qui pourrait remettre en cause la parasémie serait l'existence au sein de la langue de signes disposant d'une certaine autonomie, c'est-à-dire étymologiquement, de signes dictant à eux-mêmes la loi qui les régit. Or Saussure n'a-t-il pas précédemment indiqué que la particularité du signe relevant de l'onymique est d'être «assez défini en luimême pour échapper à loi générale du signe»? La question du nom propre est bien à l'œuvre dans le surgissement $\mathrm{du}$ concept de parasème. Lorsque dans les Cours de linguistique générale, Saussure développera les idées dont l'intuition de la parasémie constituait une première ébauche, les doutes relatifs au nom propres ne seront pas formulés. Quant à la spécificité de l'onymique dans la sémiologie, elle ne sera plus mentionnée, ni davantage analysée dans les écrits de linguistique du maître genevois.

Nous n'avons voulu ni ignorer les ambiguïtés ni résoudre les contradictions que la pensée saussurienne, dans la singularité de ses manifestations, recèle. En ce qui concerne le nom propre, force est de constater ce qui sépare son traitement dans les Notes Item et son absence du champ de la linguistique générale dans les Cours. Faut-il nécessairement supposer une évolution de la position saussurienne sur cette question? N'est-ce pas plutôt que l'ambiguïté consubstantielle de ce signe particulier n'avait pas sa place dans l'exposé à des étudiants des quelques principes positifs d'une science du langage ? Il est néanmoins manifeste qu'à travers les écrits autographes, les légendes et les anagrammes, Saussure a entrepris de percer le fonctionnement singulier du nom propre.

Au terme de ce parcours, il apparaît que Whitney, Bréal et Saussure, chacun à leur façon, se sont confrontés à la question du sens de ces noms. Si les deux premiers, nous l'avons vu, s'accordent à concevoir entre noms communs et noms propres une différence de degré, la question du sens de ces noms les oppose radicalement. Chez Whitney, le nom propre constitue un élément linguistique primitif, à la fois dans l'apparition du langage humain et dans l'apprentissage de la langue maternelle, auquel est conféré une charge sémantique minimale. Bréal, quant à lui, rend la signification d'un nom inversement proportionnelle à son extension référentielle, démontrant ainsi que les noms propres sont «les plus significatifs de tous ». Chacune de ces positions traduit un présupposé épistémologique de son auteur: une conception nomenclaturiste de la langue pour le premier, l'influence de la grammaire de Port-Royal pour le second. Chez Saussure, c'est dans les Notes Item, où il répudie et l'adamisme de Whitney et la sémantique bréalienne, que la question du nom propre trouve son traitement le plus abouti. Et c'est cette question qui l'amène à consigner pour la première fois, avec la notion de parasème, une conception de la signification comme phénomène contrastif issu de ce système de valeur que constitue un état de langue.

Au tournant des $\mathrm{XIX}^{\mathrm{e}}$ et $\mathrm{XX}^{\mathrm{e}}$ siècles, période qui a vu l'élaboration progressive d'un point de vue général sur les langues ainsi que la naissance d'une nouvelle discipline, la sémantique, le rôle du nom propre chez trois de ses plus grands linguistes ne manque pas d'étonner. Et l'on songe que la problématique du nom propre n'est peut-être pas étrangère à l'une des inventions linguistiques capitales de ces années, le signifié.

\section{Bibliographie}

Arnaud, A. et Lancelot, C. $(1660$; 1968). Grammaire générale et raisonnée de Port-Royal. Genève: Slatkine Reprints.

Arnauld, A. et Nicole, P. (1662 ; 1992). La logique ou l'art de penser. Paris : Gallimard.

Auroux, S., Deschamps, J., Kouloughli, D. (2004). La philosophie du langage. Paris: Presses Universitaires de France.

Baratin, M. et Desbordes, F. (1981). L'analyse linguistique dans l'antiquité classique. 1 les théories. Paris: Klincksieck.

Baratin, M. (2006). A propos du nom propre dans l'Antiquité : quelques points qui ont fait débat. Corpus, 50, 229237. 
Benveniste, E. (1966). Problèmes de linguistique générale. Paris : Gallimard.

Bergounioux, G. (éd.) (2000). Bréal et le sens de la sémantique. Orléans : Presses Universitaires d'Orléans.

Bouquet, S. (1992). La sémiologie linguistique de Saussure : une théorie paradoxale de la référence ? Langages, 107, 84-95.

Bréal, M. (1897 ; 1982). Essai de sémantique : science des significations. Brionne : Gérard Monfort.

Constantin E. (2005) Linguistique générale, Cours de M. le professeur de Saussure, 1910-1911. Ed. par Mejia C. Cahiers Ferdinand de Saussure, 58, 83-289.

Darmesteter, A. (1887). La vie des mots étudiée dans leur signification. Paris : Delagrave.

Delesalle, S. et Chevalier, J.-C. (1986). La linguistique, la grammaire et l'école. 1750-1914. Paris : Armand Colin.

Desmet, P. (1994). La linguistique naturaliste en France (1867-1922). Nature, origine et évolution du langage. Louvain : Peeters.

Frege, G. (1892 ; 1971). Ecrits logiques et philosophiques. Paris : Seuil.

Gary-Prieur, M.-N. (1991). Le nom propre constitue-t-il une catégorie linguistique ? Langue française, 92, 4-25.

Gary-Prieur, M.-N. (1994). Grammaire du nom propre. Paris : Presses Universitaires de France.

Joseph, J. E. (1988). Saussure's meeting with Whitney, Berlin, 1879. Cahiers Ferdinand de Saussure, 42, 205-213.

Kleiber, G. (1981). Problèmes de référence : descriptions définies et noms propres. Paris : Klincksieck.

Lallot, J. (2007). L'invention du nom propre dans la tradition grecque ancienne. Lalies, 27, 233-246.

Leibniz, G. W. (1710 ; 2002). Bref essai sur l'origine des peuples déduite principalement des indications fournies par les langues. Paris : Seuil.

Leroy, S. et Muni Toke, V. (2007). Une date dans la description linguistique du nom propre : l'Essai de grammaire de la langue française de Damourette et Pichon. Lalies, 27, 115-190.

Mejia C. (1999) L'aposème, unité de parole. Cahiers Ferdinand de Saussure 52, 237-253.

Mill, J. S. (1843 ; 1988). Système de logique déductive et inductive. Liège-Bruxelles : Pierre Mardaga.

Molino J. (1982) Le nom propre dans la langue. Langages, 66, 5-20.

Müller, M. (1898 ; 2002). Nouvelles études de mythologie. Paris : Robert Laffont.

Noailly M. (éd.) (1995) Nom propre et Nomination. Paris : Klincksieck.

Normand, C. (éd.) (1978). Saussure et la linguistique pré-saussurienne. Langages, 49, Paris : Larousse.

Russell, B. (1956) Logic and Knowledge. Londres : Georges Allen and Unwin.

Saussure, F. de (1916) Cours de linguistique générale. Ed. par Bally C. et Séchehaye A.. Genève : Payot.

Saussure, F. de (1922). Recueil des publications scientifiques de Ferdinand de Saussure. Ed. par Bally C. et Gautier L.. Genève : Payot.

Saussure, F. de (1967-1974). Cours de linguistique générale. Ed. critique par Engler R.. Wiesbaden: Otto Harrassowitz.

Saussure, F. de (1986). Le Leggende Germaniche. Ed. par Marinetti A. et Meli M.. Este : Zielo.

Saussure, F. de (2002). Ecrits de linguistique générale. Ed. par Engler R. et Bouquet S.. Paris : Gallimard.

Whitney, W. D. (1875). La vie du langage. Paris : Germer Baillière et Cie.

\footnotetext{
${ }^{1}$ Whitney $(1875 ; 1877)$, p. 1.

${ }^{2}$ Idem, p. 22.
} 


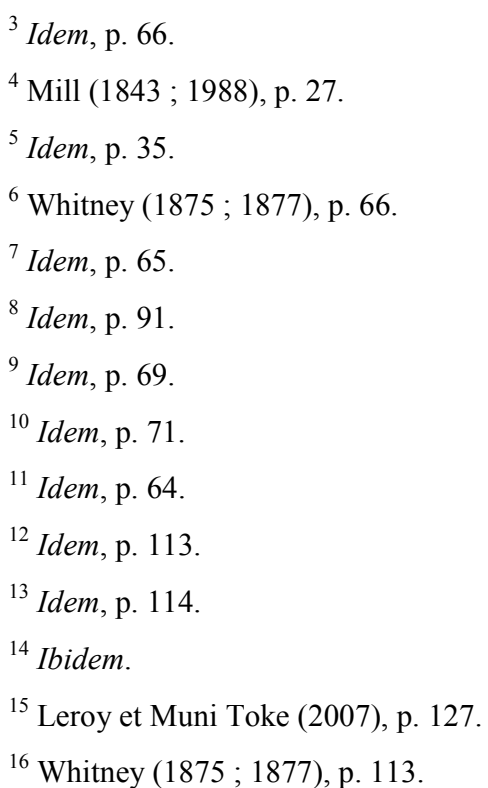

${ }^{17}$ Cf. Idem, p. 114 : «D'abord il y a toujours une idée qui précède le mot. [...] Cela est si évident que personne ne songe à le nier. Essayer de le faire, ce serait prétendre qu'un objet nouveau ne peut être connu avant que d'avoir eu un nom, ou que l'enfant n'est pas né avant que les fonts baptismaux ne l'aient vu. »

${ }^{18}$ Les plus illustres représentants de cette école naturaliste sont sans conteste Auguste Schleicher et Max Müller. Piet Desmet (1994) a mis en lumière l'existence, autour d'Abel Hovelacque, d'une école de linguistique naturaliste en France contemporaine de Michel Bréal.

${ }^{19}$ Bréal $(1897$; 1982), p. 3.

${ }^{20}$ Cf. les titres respectifs de ces quatre chapitres: «La restriction de sens », «élargissement de sens », «la métaphore », « des mots abstraits et de l'épaississement du sens ».

${ }^{21}$ Idem, p. 182.

${ }^{22}$ Idem, p. 317

${ }^{23}$ Idem, p. 182.

${ }^{24}$ Sur ce débat et, tout particulièrement, sur l'opposition dans l'antiquité entre stoïciens, pour qui les noms propres constituent une classe grammaticale distincte des appellatifs, et péripatéticiens, partisans d'une inclusion des noms propres dans une catégorie générale du nom, nous renvoyons aux travaux de Lallot (2007) et Baratin (1981), (2006).

${ }^{25}$ Cf. les affirmations respectives de Leibniz $(1710$; 2002), p. 171 - «Les traces les plus archaïques des langues subsistent dans les noms des fleuves et des forêts qui survivent généralement aux changements de population » - et Müller $(1898 ; 2002)$, p. 451 : «C'est un fait facile à constater sur les noms propres de lieu : ils violent souvent les lois phonétiques les plus sacrées. »

${ }^{26}$ Cf. Delesalle et Chevalier (1986), p. 285 seq. pour qui le refus radical de l'étymologie, alors qu'elle est encore centrale chez Darmesteter, et la primauté de l'analogie sont les deux fondements de la sémantique bréalienne.

${ }^{27}$ Bréal $(1897 ; 1982)$, p. 182.

${ }^{28}$ Ibidem.

${ }^{29}$ Voici comment sont définis ces concepts dans la Logique de Port-Royal : « J'appelle compréhension de l'idée, les attributs qu'elle enferme en soi, et qu'on ne peut lui enlever sans la détruire [...]. J'appelle étendue de l'idée les sujets à qui cette idée convient. » (Arnauld et Nicole (1662;1992), p. 52)

${ }^{30}$ Auroux, Deschamps et Kouloughli (2004), p. 145. 
${ }^{31}$ Cf. par exemple la Grammaire générale et raisonnée de Port-Royal où noms propres et appellatifs sont présentés comme les deux faces d'une même unité grammaticale (Arnaud et Lancelot (1660; 1968), p. 51 seq.).

${ }^{32}$ Sans nous étendre sur ces considérations biographiques, signalons que le jeune Saussure qui préparait sa thèse de sanscrit a rencontré Whitney, auteur d'une grammaire sanscrite, à Berlin en 1879 (cf. Joseph (1988)). Quant à Bréal, c'est à lui qu'est due la nomination de Saussure à l'Ecole Pratique des Hautes Etudes au poste de maître de conférence de gothique et de vieux haut-allemand qu'il occupa de 1881 à 1891 . Leur correspondance atteste de la profonde affection qui unit les deux hommes.

${ }^{33}$ Cf. par exemple Gary-Prieur (1991), p. 12: "On voit que le Nom Propre est d'emblée déclaré "isolé » et « inanalysable » dans le système des signes qu'est la langue. Et cela, probablement (Saussure ne s'en explique nulle part), parce qu'un signe " sans signifié » ne peut être qu'un objet extérieur au système ». Voir également Gary-Prieur (1994), p. 3 seq. et Leroy et Muni Toke (2007), p.127-129.

${ }^{34}$ Saussure (1916), p. 237.

${ }^{35}$ Idem, p. 226.

${ }^{36}$ On aura évidemment noté à cet égard l'implacable itération de l'adjectif « aucun ».

37 Cf. «Le nom de la ville d'Oron à l'époque romaine», «Origine de quelques noms de lieux de la région genevoise », «Les Burgondes et la langue burgonde en pays roman » et « Le nom du Jura » (Saussure (1922), p. 604607).

${ }^{38}$ Saussure (1986), p. 31.

${ }^{39}$ Idem, p. 142.

${ }^{40}$ Noms de dieux qu'il s'agit de «river» au texte dans le cadre de la poésie religieuse, nom du destinataire de l'œuvre ou signature de l'auteur: la nature des noms varie suivant les fonctions que Saussure envisage pour l'anagramme.

${ }^{41}$ Cf. dans le Cours (Saussure (1916), p. 34, 97, 158) et dans les notes manuscrites (Saussure (1967-1974), désormais abrégé CLG/E : CLG/E, 3299, 3336.

${ }^{42} \mathrm{CLG} / \mathrm{E}, 3299$.

${ }^{43}$ Benveniste (1966), p. 50.

${ }^{44}$ Idem, p. 54.

${ }^{45}$ Bouquet (1992).

${ }^{46} \mathrm{CLG} / \mathrm{E}, 3308$.

${ }^{47}$ Bréal $(1897 ; 1982)$, p. 177.

${ }^{48} \mathrm{CLG} / \mathrm{E}, 3312$.

49 Dans un texte développant une idée similaire, d'autres exemples sont utilisés : «C'est un accident quand le signe linguistique se trouve correspondre à un objet défini pour les sens comme un cheval, le feu, le soleil, plutôt qu'à une idée comme $\theta \eta \kappa \varepsilon$ 'il posa'〉. Quelle que soit l'importance de ce cas, il n'y a aucune raison 〈évidente〉, bien au contraire, de le prendre comme type du langage.» (CLG/E, 3299.) Remarquons que ce sont pourtant deux de ces cas particuliers ou accidentels, arbre et cheval, que Saussure emploiera dans le troisième cours pour exposer sa théorie du signe.

${ }^{50}$ Saussure (2002), p. 96-97.

${ }^{51} \mathrm{CLG} / \mathrm{E}, 3311.1$.

${ }^{52} \mathrm{CLG} / \mathrm{E}, 3314.8$. Pour une étude détaillée de cette notion nous renvoyons à Mejia (1999).

${ }^{53}$ Cf. par exemple : «Item. † Ce qu'on appelle signification [...] ne peut jamais être dégagée de manière à devenir elle-même un objet de recherche, ou d'observation. Entendons-nous bien : elle peut devenir dans une certaine mesure un tel objet de recherche et d'observation à la condition qu'on en revienne sans cesse au sème. » CLG/E, 3320.3. 
Histoire, épistémologie, réflexivité DOI $10.1051 / \mathrm{cmlf0} 040$

\footnotetext{
${ }^{54}$ CLG/E, 3313.2.

${ }^{55}$ CLG/E, 1857 ; Constantin (2005), p.282.

${ }^{56}$ CLG/E, 1861 ; Idem, p. 282-283.

${ }^{57}$ CLG/E, 1909 : Idem, p. 288.

${ }^{58}$ CLG/E, 1357 ; Idem, p. 260.
} 